\section{Diagnosing eosinophilic esophagitis}

\section{Diagnosticando la esofagitis eosinofílica}

To the Editor,

We recently read this interesting article in the journal: Esofagitis eosinofílica: diagnóstico y manejo${ }^{1}$. After its description, eosinophilic esophagitis (EoE) has become an epidemiological and editorial phenomenon $^{2}$. We would like to highlight several points, including those related to the definition of EoE. This has gone through some stages; the first diagnostic guide was done in 2007 and was updated in 2011. The last international consensus for the diagnostic criteria of EoE was carried out in $2018^{3}$ and indicates that several aspects are necessary: - the presence of symptoms of esophageal dysfunction, - in esophageal histology the presence of $\geq 15$ eosinophils per high-power field (hpf). Eosinophilic infiltration should be isolated to the esophagus. Other aspects, such as endoscopic findings and atopic comorbidities increase the diagnostic suspicion, but they are not essential and may be absent. In theTop of Form article published ${ }^{1}$, the diseases that cause or may contribute to esophageal eosinophilia are listed, and it is stated that according to the 2013 guidelines, these should be excluded. However, in the 2018 consensus, in addition to including PPI-responsive esophageal eosinophilia within the spectrum of EoE, it is stated that the diseases listed as conditions associated with esophageal eosinophilia should be evaluated rather than require their exclusion, since they can coexist in some cases, such as gastroesophageal reflux disease (GERD), which is one of the most complex differential diagnoses ${ }^{3}$. The esophageal motility disorders can also be clinically difficult to differentiate and an overlap has been described ${ }^{4}$. Other causes of esophageal eosinophi- 
lia are rare or have distinctive clinical features. In this regard, it is useful to take gastric and duodenal biopsy samples, which are always recommended in pediatric guidelines, and in adults, symptoms and endoscopic findings must be taken into account to obtain them. Since EoE is the same disease in children as in adults, the diagnostic criteria are applicable to all age groups ${ }^{3}$.

Regarding epidemiology, we would like to point out that although the authors of the $\operatorname{article}^{1}$ did not find important epidemiological studies of EoE in adults within the Latin American region, there are studies in the pediatric population carried out by the Latin American Society for Pediatric Gastroenterology, Hepatology and Nutrition (LASPGHAN). A multicenter study (36 centers in 10 Latin American countries) determined a general prevalence rate of $3.69 \times 1000$. In those submitted to endoscopy, it was $26 \times 1000$. Although not all the countries of the region are represented, it is interesting to note that the prevalence showed significant variability between the participating countries and centers. Mexico, Central America (Salvador), and the Andean region (Peru and Ecuador) were identified with low prevalence. The North Caribbean (Venezuela) and the Southern Cone (Uruguay, Argentina, and Chile) presented intermediate prevalence; the highest was reported in Brazil, followed by Colombia. When analyzing the epidemiology of the region, it is suggested to take into account that Latin America is a very heterogeneous area, with great ethnic and socioeconomic development variability of the different countries, this can influence the prevalence. EoE occurs more frequently in urban populations with higher socioeconomic status, which may also be reflected in some countries and sectors of the population in Latin America. The uneven development of the specialty of gastroenterology and digestive endoscopy is also involved ${ }^{2}$.

Regarding the clinical picture, in pediatric age it may be more complex to have a diagnostic suspicion based on symptoms, since, especially in younger children, symptoms may be more nonspecific. The spectrum of symptoms is also greater at a younger age and more difficult to define since they depend on the interpretation of their caregivers and require a questioning directed by the doctor. Thus, in infants and younger children, symptoms can be vague or ambiguous, includes feeding difficulties like aversion, rejection or intolerance to food, choking with meals, vomiting, regurgitation, weight loss, failure to thrive, cough, and sleep disturbance. In school children, dysphagia can already be reported in the directed questioning, food impaction, choking /gagging with coarse textures of food, abdominal or chest pain, odynophagia, as well as symptoms compatible with GERD such as regurgitation and heartburn, vomiting, nausea, sleep disturbance, and decreased appetite. In adolescents and adults, the most characteristic symptoms of EoE are reported more frequently, such as dysphagia and impaction of food, in addition to food avoidance, intractable heartburn, regurgitation, and retrosternal or chest pain ${ }^{4}$. Therefore, in the presence of esophageal symptoms, always take a biopsy sample of the esophagus in distal and middle/ proximal thirds to be evaluated.

Finally, due to its use in several studies, we mention scores that can favor the diagnosis and follow-up of EoE, and above all, scientific research, since the findings take a quantitative value. In the EoE Endoscopic Reference Score described by Hirano et $\mathrm{al}^{5}$, the findings are quantified according to severity, it is called EREFS by its english acronym: Edema $0-2$ points, Rings $0-3$ points, Exudates $0-2$ points, longitudinal Furrows $0-2$ points, Stenosis $0-1$ point. With a maximum of 10 points, the higher values represent greater endoscopic severity. Although the findings of mucosal friability, crepe paper mucosa in EoE have also been described, these are not included in the endoscopic score. Recently there have also been several publications on a histological score (EoE Histology Scoring System: EoE HSS) ${ }^{6}$, with studies that suggest its superiority compared to the peak of the eosinophil count. This incorporates items like eosinophilic inflammation, basal zone hyperplasia, eosinophil abscess, eosinophil surface layering, dilated intercellular space, surface epithelial alteration, dyskeratotic epithelial cells and lamina propria fibrosis. This score has not yet become generalized in routine diagnostic practice; perhaps more prospective studies are necessary before its general use.

Sarah Esther Díaz-Olival,
Idalmis Aguilera-Matos ${ }^{1}$, Angel A. Escobedo'
,
Oscar M. Villa-Jiménez ${ }^{3}$, Leonor Chacin-Bonilla
${ }^{4}$ Departamento de Gastroenterología Pediátrica,
Instituto de Gastroenterología, La Habana, Cuba.
${ }^{2}$ Departamento de Epidemiología, Instituto de
Gastroenterología, La Habana, Cuba.
${ }^{3}$ Departamento de Investigaciones, Instituto de
Gastroenterología, La Habana, Cuba.
${ }^{4}$ Sección de Parasitología, Instituto de Investiga-
ciones Clínicas, Universidad del Zulia, Maracaibo,
Venezuela.

\section{References}

1. Ballart MJ, Monrroy H, Iruretagoyena M, Parada A, Torres J, Espino A. Esofagitis eosinofílica: Diagnóstico y manejo. Rev Med Chile 2020; 148: 831-41.

2. Pierre R, Vieira M, Vázquez R, Ninomiya I, Messere G, Daza W, et al. Prevalencia de esofagitis eosinofílica: estudio multicéntrico en población pediátrica evaluada en 36 centros de gastroenterología de América Latina. Revista de Gastroenterología de México. 2019; 84 (4):427-33.

3. Dellon ES, Liacouras CA, Molina-Infante J, Furuta GT, 
Spergel JM, Zevit N, et al. Updated international consensus diagnostic criteria for eosinophilic esophagitis: proceedings of the AGREE conference. Gastroenterology. 2018; 155 (4): 1022-33. e10.

4. Díaz-Oliva SE, Aguilera-Matos I, Villa Jiménez OM, Escobedo A. Oesophageal eosinophilia and oesophageal diseases in children: are the limits clear? BMJ Paediatrics Open 2020; 4: e000680.

5. Hirano I, Moy N, Heckman MG, Thomas CS, Gonsalves $\mathrm{N}$, Achem SR. Endoscopic assessment of the oesophageal features of eosinophilic oesophagitis: validation of a novel classification and grading system. Gut 2013; 62: 489-95.
6. Collins MH, Martin LJ, Alexander ES, Boyd JT, Sheridan $\mathrm{R}, \mathrm{He} \mathrm{H}$, et al. Newly developed and validated eosinophilic esophagitis histology scoring system and evidence that it outperforms peak eosinophil count for disease diagnosis and monitoring. Dis Esophagus. 2017; 30 (3): 1.

Corresponding Author:

Dra. Sarah Esther Díaz-Oliva

Reforma 11205

Reparto Antonio Maceo, Cerro, La Habana, Cuba.

sarahediazo@gmail.com 